\title{
Challenges and possible solutions in dental practice during and post COVID-19
}

\author{
Jayachandra Bhumireddy ${ }^{1}$ (D) Sreekanth Kumar Mallineni ${ }^{2,3}$ (D) $\cdot$ Sivakumar Nuvvula $^{2}$ (i)
}

Received: 1 June 2020 / Accepted: 23 September 2020 / Published online: 7 October 2020

(C) Springer-Verlag GmbH Germany, part of Springer Nature 2020

\begin{abstract}
The pandemic outbreak of coronavirus disease (COVID-19) created a significant impact on the oral healthcare profession, and dentists are reported to have a higher risk of transmission. Aerosols and droplets are considered as primary reasons for the transmission of this infectious disease. Quality dental treatment may not be possible without the interference of aerosols in daily dental practice. However, dental societies and healthcare communities around the globe developed various protocols and algorithms with standard infection control protocols. Nevertheless, many unanswerable questions strike in dentist minds to start the dental practice during or post COVID-19 pandemic. Hence, this review focuses on the common issues that raises in daily clinical practice and possible solutions.
\end{abstract}

Keywords Aerosols $\cdot$ COVID-19 $\cdot$ Dentistry $\cdot$ Challenges $\cdot$ Recommendations

\section{Introduction}

The recent pandemic outbreak of pneumonia that has spread worldwide was caused by a novel coronavirus (COVID-19) (Peng et al. 2020). This virus was contagious and spread through respiratory droplets via coughing, sneezing, and physical contact (Izzetti et al. 2020). In order to prevent faster transmission and mortality, the lockdown was preferred globally. The current scenario of global lockdown has created panic in the minds of the oral healthcare personnel as to whether to continue the practice by overcoming the challenges or to remain benign until the world restores to normalcy. Oral health professionals are vulnerable and have a higher risk of the transmission

Responsible Editor: Lotfi Aleya

Sreekanth Kumar Mallineni

drmallineni@gmail.com; s.mallineni@mu.edu.sa

1 Pediatric and Preventive Dentistry, Saraswati Dhanwantri Dental College and Hospital \& Post Graduate Research Institute, Parbhani, Maharastra, India

2 Department of Pediatric and Preventive Dentistry, Narayana Dental College and Hospital, Nellore, India

3 Pediatric Dentistry, Department of Preventive Dental Science, College of Dentistry, Majmaah University, Majmaah 11952, Saudi Arabia of this COVID-19 (Meng et al. 2020). The major drawback is that dentistry involves the production of contaminated aerosols, which continue to be a major source of cross infection. This paper addresses information on few challenges, of which some of them may require additional research and time.

\section{Challenge 1: Does coronavirus spread only through patients with active symptoms?}

No. It can spread through asymptomatic, symptomatic, and even pre-symptomatic transmission. It can also spread from patients in the convalescence stage (Izzetti et al. 2020). It can also spread through contact transmission. Hence, it is tough to identify super spreaders and limit them in general dental practice, even if the dental operatory has restricted to a dental emergency.

\section{Challenge 2: Is the dental team well equipped to deal with COVID-19?}

This new pandemic is a sudden outburst to the world with the non-availability of vaccine or specific treatment regimen to date (Padron-Regalado 2020). The current knowledge is inadequate to safeguard the dental team from the risk of transmitting the virus to others. There were many reported deaths of 
healthcare workers fighting to save patients even with appropriate use of PPE and personal disinfection (Zhan et al. 2020).

\section{Challenge 3: Can I run my dental practice after the COVID-19 pandemic as I run it before?}

No. Without sufficient knowledge on COVID-19, running a dental practice is akin to creating a hot spot for virus transmission. Additional changes are needed in the dental setting such as wide reception areas with social distancing between patients, employees, telephone triage, scheduling accurate necessary appointments, high level of disinfection, extra-oral high volume of the suction system, negative pressure rooms, strict sterilization protocols, strong adherence to the use of N-95 masks, and PPE (Meng et al. 2020; Mallineni et al. 2020). There should be a strong promotion towards hand hygiene by the dental team and patients before entering/leaving the dental office.

\section{Challenge 4: Does my current knowledge adequate to practice?}

Not sure. As the data is rapidly evolving, additional training and update are needed to be compliant. It is essential to teach and share the updated recommendations to the entire team (Padron-Regalado 2020). The knowledge of proper donning and doffing of PPE is crucial to the whole dental team.

\section{Challenge 5: Can I be cost-effective to my patients?}

It may not be possible. As the cost of PPE is high and needs to be changed to every patient, along with additional disinfection measures and a limited number of appointments per day, the cost of dental treatment may increase and burden our patients (Ferneini 2020).

\section{Challenge 6: Are dentists really at high risk when compared with other healthcare workers?}

Yes. Dental practitioners are potentially at a higher risk. Because coronavirus has a high affinity to ACE2 receptors, which are highly expressed in salivary glands than respiratory mucosa, saliva acts as the primary reservoir of droplet transmission (Xu et al. 2020). Droplet, splash, and spatter production is high during dental procedures with the use of high- and low-speed handpiece, 3-way syringe, and an ultrasonic scaler
(Peng et al. 2020). The droplets with the virus may remain alive for few hours to days depending on the surface and may remain suspended in the air for a few hours, depending on the surrounding atmosphere (Meng et al. 2020).

\section{Challenge 7: Is managing saliva a significant challenge for the dentist?}

Yes. Saliva acts as a significant reservoir of virus load, and droplets constitute a substantial source of airborne and contact transmission of novel coronavirus (Xu et al. 2020). The size of the salivary droplets determines the range of transmission (diameter $<10 \mu \mathrm{m}$ has the potential for long-range transmission). One cough may generate three thousand droplets, and a sneeze may generate 40,000 droplets reaching several meters in the air, suggesting the need to be conscious, vigilant during practice (Xu et al. 2020). Aerosols generated during dental treatment are mixed with saliva, blood, and various pathogens. They contaminate the air and inanimate surroundings in the working area and may remain suspended in the air for prolonged periods. In the view of this dangerous, potentially infective arena, the use of disposable PPE; face shields; goggles; surface disinfection with $0.1 \%$ sodium hypochlorite, 62 $71 \%$ ethanol, or $0.5 \%$ hydrogen peroxide; and well-ventilated treatment rooms remains crucial (Ather et al. 2020).

\section{Challenge 8: Can I defer treatment to a patient during or post COVID-19?}

Ethical issues of treating a patient during or post pandemic mostly depend on the concerned country or state authority regulations. Most of the dental bodies recommend emergency and urgent dental care (Mallineni et al. 2020). Social distancing may not be possible during emergency care. It may carry the risk of being infected to the entire dental team, which may, in turn, be a source of infection to the community. The moral decision to treat a patient under a dental emergency is in line with esteemed professionalism and professional assurance (Mallineni et al. 2020). Conscious and careful modification of treatment consent forms highlighting the risk of virus transmission, self-responsible monitoring, and alarming dentist if any symptoms arise post-treatment is necessary to curtail legal and professional indemnity.

Although various infection control protocols had have been recommended to avoid transmission of COVID-19 disease in dental practice (Meng et al. 2020; Peng et al. 2020), it has been reported that COVID-19 can be spread through direct and indirect communication, predominantly through respiratory droplets, and spatter from saliva and blood (Meng et al. 2020; Mallineni et al. 2020) and this makes dentists in potentially high 
risk. Most of the dental procedures involve aerosols, which increase the chances of the cross contamination of acute respiratory infections (Meng et al. 2020; Peng et al. 2020). Besides that, the dental operatory has a more number of contaminated surfaces which include the dental instruments, the spittoon, doctor stools, and dental chairs and their handles, and these are potential sources for transmission. However, adhering to stringent protocols like environmental surface disinfection, compulsory cough etiquette instruction, meticulous hand hygiene, pre-procedural mouth rinse, rubber dam isolation, use of appropriate particulate respirator masks like certified N95, European Standard Filtering Face Piece 2 (EU FFP2), or European Standard Filtering Face Piece 3 (EU FFP3) respirators in case of dental emergencies (Mallineni et al. 2020). The use of inexpensive highvolume evacuator (HVE) and the expensive highefficiency particulate arrestor (HEPA) filters is highly recommended during and post COVID-19 pandemic (Ge et al. 2020). Nevertheless, dentists cannot avoid the production of aerosols while providing quality dental treatment, and they can only limit their production by following specific guidelines on urgent/emergency dental care and adhering to stringent protocols. The recent COVID-19 pandemic outbreak has impaired socioeconomic and ethnic disproportions and will unquestionably deteriorate dental practice globally. Currently, it is very essential to be more approachable to the oral healthcare needs of people and prioritize oral care for groups with high demand. It is more important to introduce virtual consultation using the teledentistry model for the benefit of society and oral healthcare professionals. The present emergency has evidently exposed dental practitioners, and the dental profession could be incorporated into the broader system, and the challenge ahead is to be outlined the clinical roles of dentists in a more cohesive model of care.

Acknowledgments The authors would like to thank the Deanship of Scientific Research at Majmaah University for supporting this work with Project No (R1442-XX).
Authors' contributions Project administration: M.S.K; writing-original draft: J.C.B. and M.S.K; writing-review and editing: N.S.K. and M.S.K; approval of the manuscript for publication: J.C.B., M.S.K., and N.S.K.

Availability of data and materials Not applicable.

\section{Compliance with ethical standards}

Conflict of interest The authors declare that they have no conflict of interest.

\section{References}

Ather A, Patel B, Ruparel N, Diogenes A, Hargreaves KM (2020) Coronavirus disease 19 (COVID-19): implications for clinical dental care. J Endod 46(5):584-595

Ferneini E (2020) The financial impact of Covid-19 on our practice. J Oral Maxillofac Surg 78:1047-1048. https://doi.org/10.1016/j.joms. 2020.03.045

Ge XY, Yang LM, Xia JJ, Fu XH, Zhang YZ (2020) Possible aerosol transmission of COVID-19 and special precautions in dentistry. J Zhejiang Univ Sci B 21:361-368. https://doi.org/10.1631/jzus. B2010010

Izzetti R, Nisi M, Gabriele M, Graziani F (2020) COVID-19 transmission in dental practice: brief review of preventive measures in Italy. J Dent Res 99:1030-1038. https://doi.org/10.1177/ 0022034520920580

Mallineni SK, Innes N, Raggio D, Araujo M, Robertson M, Jayaraman J (2020) Coronavirus disease (COVID-19): characteristics in children and considerations for dentists providing their care. Int J Paediatr Dent 30:245-250

Meng L, Hua F, Bian Z (2020) Coronavirus disease 2019 (COVID-19): emerging and future challenges for dental and oral medicine. J Dent Res 99(5):481-487

Padron-Regalado E (2020) Vaccines for SARS-CoV-2: lessons from other coronavirus strains. Infect Dis Ther 9(Apr 23):255-274. https:// doi.org/10.1007/s40121-020-00300-X

Peng X, Xu X, Li Y, Cheng L, Zhou X, Ren B (2020) Transmission routes of 2019-nCoV and controls in dental practice. Int J Oral Sci 12(1):9

Xu R, Cui B, Duan X, Zhang P, Zhou X, Yuan Q (2020) Saliva: potential diagnostic value and transmission of 2019-nCoV. Int J Oral Sci 12(1):11. https://doi.org/10.1038/s41368-020-0080-Z

Zhan M, Qin Y, Xue X, Zhu S (2020) Death from Covid-19 of 23 health care workers in China. N Engl J Med. https://doi.org/10.1056/ nejmc 2005696

Publisher's note Springer Nature remains neutral with regard to jurisdictional claims in published maps and institutional affiliations. 